\title{
Cell Cycle Regulatory CCND1 G870A Gene Polymorphism and Periodontitis-Induced Oral Cancer: A Risk Analysis
}

\author{
Mahvish Khan ${ }^{1}$, Saif Khan ${ }^{2 *}$, Raju K. Mandal ${ }^{3}$, Hari S. Mahto ${ }^{4}$, Mohtashim \\ Lohani $^{5}$, Saheem Ahmad ${ }^{6}$, Subuhi Sherwani ${ }^{7}$, Suresh B. Jandrajupalli ${ }^{8}$, Shafiul \\ Haque $^{3}$
}

1. Department of Biology, College of Science,, University of Ha'il, Ha'il-2440, Saudi Arabia

2. Department of Basic Dental and Medical Sciences, College of Dentistry, Ha'il University, Ha'il-2440, Saudi Arabia

3. Research and Scientific Studies Unit, College of Nursing \& Allied Health Sciences, Jazan University,

$$
\text { Jazan-45142, Saudi Arabia }
$$

4. Centre for Life Sciences, Central University of Jharkhand, Ranchi-835205, Jharkhand, India

5. Department of Emergency Medical Services, College of Applied Medical Sciences, Jazan University,

$$
\text { Jazan-45142, Saudi Arabia }
$$

6. Department of Clinical Laboratory Sciences, College of Applied Medical Sciences, University of Ha'il, Ha'il-2440, Saudi Arabia

7. Department of Biology, College of Science, University of Ha 'il, Ha 'il-2440, Saudi Arabia

8. Department of Preventive Dentistry, College of Dentistry, University of Ha'il, Ha 'il-2440, Saudi Arabia

\begin{abstract}
Strong association has been recently observed between periodontitis/gingivitis and Oral squamous cell carcinoma (OSCC). A high incidence of oral cancer has been reported in the case of chronic periodontitis. Recently Cell cycle regulatory /Senescence genes have been associated with Gingivitis/ Periodontitis susceptibility. Cyclin D1 is one such cell cycle regulatory gene. Several findings have reported that Cyclin D1 (CCND1) G870A Single nucleotide polymorphism is associated with oral cancer (OC) risk, but yielded inconsistent data across different studies. This meta-analysis explores the precise relationship between CCND1 G870A polymorphism and OC risk. PubMed (Medline), EMBASE, \& Google Scholar databases were searched for eligible studies and pooled odds ratios (ORs) and $95 \%$ confidence intervals (CI) were calculated. Newcastle-Ottawa analysis was done for selected articles qual-
\end{abstract}

\footnotetext{
* Corresponding author: Saif Khan, Department of Basic Dental and Medical Sciences, College of Dentistry, Ha'il University, Ha'il-2440, Saudi Arabia. E-mail: saifkhan.bio@gmail.com
} 
ity assessment, bias in publication (if any) was estimated through Funnel plots and Egger's test. Pooled analysis from eleven eligible studies suggests that CCND1 G870A polymorphism is not significantly associated with OC risk. Sub-group analysis by ethnicity failed to show any association. Sequential single study omission was performed to determine the credibility and resilience of the inferences drawn.

Keywords: CCND1 gene; periodontitis; meta-analysis; trial sequential analysis; oral cancer

Received: $1^{\text {st }}$ July 2021; Accepted: $7^{\text {th }}$ September 2021; Published: $16^{\text {th }}$ September 2021

\section{Introduction}

Soaring annual incidence (300,373/year), mortality rate $(145,238 /$ year $)$, and five-year prevalence $(702,149)$ of oral cancer characterizes it as a subtype which requires immediate response from oncology fraternity (1). Several epidemiological studies have implicated the role of periodontitis in OSCC (2-9). Recently, mechanistic insight into this association of the red complex pathogens of periodontitis and oral cancer has revealed the role of cell cycle regulatory genes in OSCC. Several cell cycle regulatory genes including CCND1 were found to be dysregulated or abnormally induced. P. gingivalis and F. nucleatum, were found to stimulate the critical molecules involved in OSCC and associated malignancies (i.e., IL-6, cyclin D1, TNF $\alpha$, MMP9, heparanase) (7-12). Here we a risk analysis based on the recently explored mechanistic link (CCND1) between periodontitis and oral cancer. Cyclin D1 (CCND1) gene is located on chromosome 11q13. CCND1 is vital for G1 to S phase transition (13). Regulation of CCND1 expression (overexpression, repression and/or inhibition) results in abnormal cell cycle progression. Repressed and/or inhibited CCND1 may result in the seizure of the cell cycle at the G1 phase. Overexpression may culminate into premature $\mathrm{S}$ transition, a crucial aspect of cancerous cell growth (14). CND1 gene is known to exhibit single nucleotide polymorphism (SNP: rs603965; codon 242, exon 4) that produces $G$ to A modification at 870 (G870A) (15). It has been suggested that the cell cycle carrying CCND1 870A allele may bypass $\mathrm{G} 1 / \mathrm{S}$ checkpoint easily and is more likely to contribute to cancer development than cells harboring opposite wild allele G (16). Since CCND1 plays a critical role in the cell cycle control, it is obvious to hypothesize that G870A SNP may affect the cell cycle ability and infer susceptibility of OC. In the recent past several case-control studies have been carried out to appraise the association between $C C N D 1$ G870A polymorphism and OC risk. However, their findings were inconclusive and even conflictive (1727). Such inconsistencies in the outcomes are possibly because of individual studies with relatively insufficient sample sizes having low statistical power to identify low penetrance genetic variant. Thus, we performed this meta-analysis with inclusion of most recent eligible published studies to solve the inconsistence of previous observations and update the more precise estimation on the relationship between the association of CCND1 G870A polymorphism and OC risk. In recent times, meta-analysis has been proven a reliable tool for establishing the genetic associations with the complex diseases by employing a quantitative approach for combining the individual results $(28)$.

\section{Materials and Methods}

\section{Identification and eligibility of studies}

All the eligible studies were identified by performing a systematic search on PubMed, Medline and Google Scholar electronic databases; last update was done in January 2020. The key words used for the search were ' $C C N D 1$ ' or ' $C y$ clin DI', 'polymorphism' and 'oral cancer' or 'oral tumor' or 'oral carcinoma' 'oral malignan- 
cy', and other single nucleotide polymorphisms (SNPs) associated keywords. In addition, we manually checked all the references found in the articles mined and found eligible for the present meta-analysis. Redundant cases found in multiple articles were identified and one with the largest sample size was selected for meta-analysis. All articles were screened for their potential eligibility.

\section{Article eligibility criteria}

Clinical Polymorphism research articles included in the present analysis were screened as per the following criteria, i.e., (i) original studies dealing with CCND1 G870A gene polymorphism and OC susceptibility; (ii) OC cases confirmed by histology or pathology; (iii) studies must be of case-control or cohort design; (iv) must provide detailed case and control frequency of all the three genotypes. Likewise, studies not included for analysis were: (i) case reports, editorial, reviews, overlapped data and animal studies; (ii) studies having none/incomplete genetic data; (iii) tumor recurrence studies and clinical observations based on treatment response; (iv) if no usable data were reported. Conflicts among authors regarding study inclusion/exclusion were resolved through discussion based on the above mentioned criteria and by involving another author acting as adjudicator.

\section{Quality assessment of the studies}

Newcastle-Ottawa scale (NOS) quality assessment was employed to evaluate the quality of all included studies of this meta-analysis (29). The publications were scored on three categories: selection, comparability, and exposure (case-control studies). These further include eight subclasses (29). This rating scale has a score range of 0 to 9 , and a star-based method is applied to weigh the quality of the articles considered in this meta-analysis. Eligible studies scoring 5 or more stars are classified as moderate to high-rank- ing quality. At least two authors were involved in quality assessment as discussed above. Disagreements on any item of the retrieved studies occurring between the investigators were resolved by open debate until the achievement of a mutual agreement. The results were reviewed by a third investigator.

\section{Statistical analysis}

We performed this meta-analysis by using allele contrast, homozygous, heterozygous, dominant, and recessive genetic models. Overall risk, ORs and $95 \%$ CIs were estimated for each included study. Heterogeneity assumption between the studies was gauged chi-square-based Q- and $\mathrm{I}^{2}$ analysis (30). The random effects model (DerSimonian and Laird method) was applied for studies having significant heterogeneity (31). Fixed effects model (Mantel-Haenszel method) was selected for studies having non-significant heterogeneity (32). Bias in publication was estimated through Funnel plots and Egger's test (33). The effect of individual study on the overall pool was estimated via sensitivity analysis, wherein a single study was removed from the pool each time to estimate the effect on the overall ORs. Significance value was set for two sided $\mathrm{p}$-value $<0.05$. Comprehensive Meta-Analysis (CMA) Version 2 software program (Biostat Inc., USA) was used to perform the meta analysis. Data entry was done by two contributing authors separately in order to avoid mistakes.

\section{Trial Sequential analysis}

Trial sequential analysis was performed (i) to adjust the threshold significance Z-boundary, or (ii) whether the quantity of the trials included in the study sufficed the minimal requirement for threshold significance. In studies where the Z-curve exceeded the Z-boundary prior to minimal required trial cases limit, no further trials were required to establish a significant relationship, if not, further trials were necessary to dis- 
cover a significant relationship between the polymorphism and associated disease. In the present study, TSA was performed through "TSA statistical tool from Copenhagen Trial Unit, Center for Clinical Intervention Research, Denmark".

\section{Results}

\section{Literature search and meta-analysis databases extraction}

Nine articles of CCND1 G870A polymorphism and $\mathrm{OC}$ association were selected. All the studies independently and carefully reviewed the literature and the information was extracted according to the pre-designed standardized data-collection form by two investigators. The characteristics collected from the included studies were: name of the first author, year of publication, ethnicity, country of origin, sample size, gene detection method, type/design of study, source of genotyping, frequency of the minor allele (MAF), and the frequency of genotypes of the cases and controls. OC therapy response analysis studies involving CCND1 SNP and mRNA analysis were rejected. The chronological strategy of selecting the germane studies used in this meta-analysis is given as PRISMA 2009 Flow Diagram (Fig 1). Table 1 and Table 2 provide the detailed characteristics including genotype distribution accompanied by MAF of the cases and controls of all the eleven studies included in the present meta-analysis.

Most of polymorphism articles ( $80 \%)$ retrieved and considered to derive this meta-analysis scored 5 stars or even more on NOS quality evaluation that indicates modest to good quality of all the studies included (Table 3).

Evaluation of publication bias and heterogeneity Funnel plot and Egger test were used to analyze the publication bias in all the studied genetic

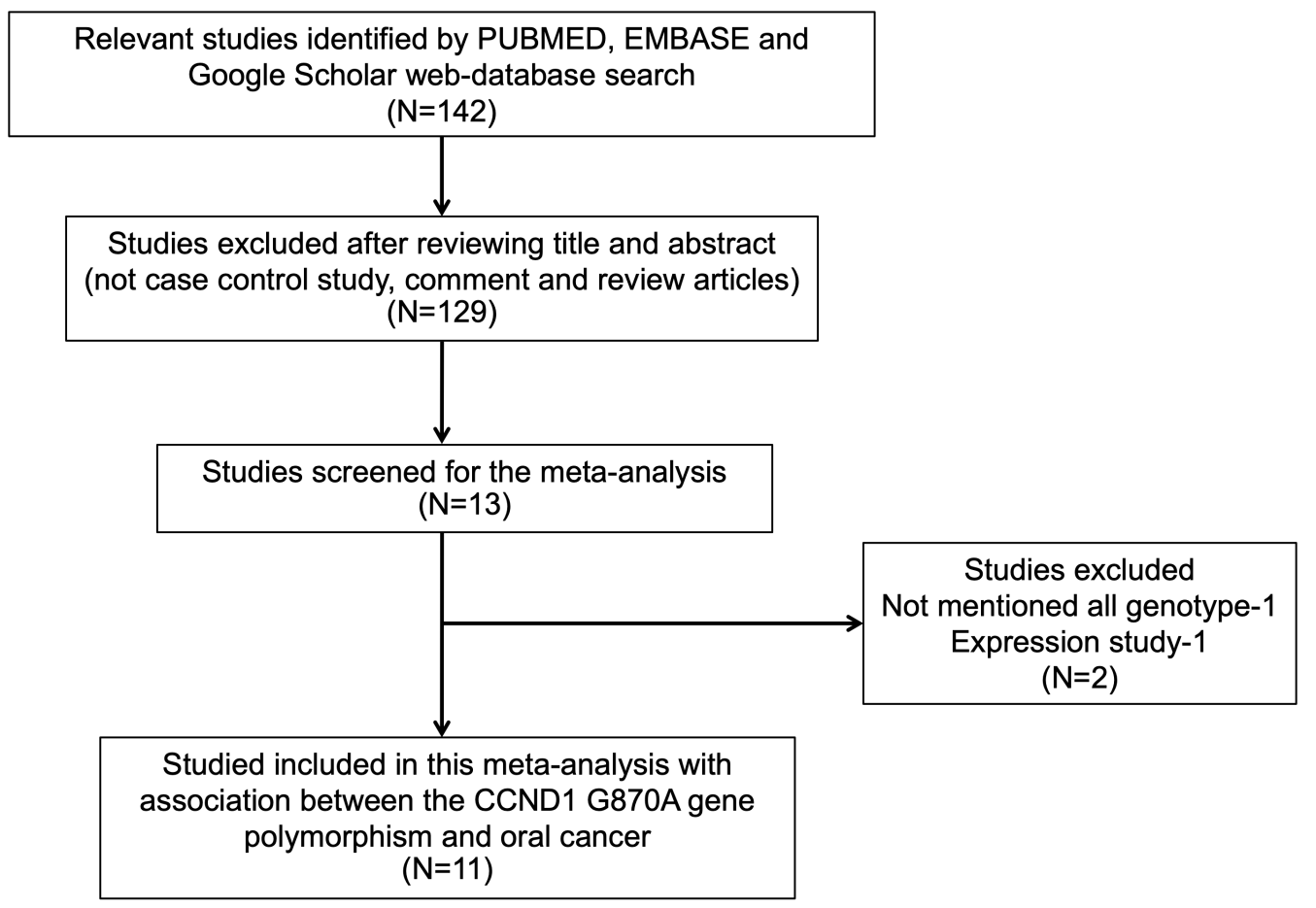

Fig. 1. PRISMA 2009 Flow-Diagram. 
Table 1. Characteristics features from articles of CCND1 G870A gene polymorphism and OC risk.

\begin{tabular}{lccccccc}
\hline $\begin{array}{l}\text { First author and } \\
\text { year }\end{array}$ & Country & Ethnicity & $\begin{array}{c}\text { Genotyping } \\
\text { method }\end{array}$ & Control & Cases & $\begin{array}{c}\text { Type of } \\
\text { study }\end{array}$ & $\begin{array}{c}\text { Source of } \\
\text { Genotyping }\end{array}$ \\
\hline Atac et al. 2014 & Germany & Caucasian & PCR-Sequencing & 102 & 83 & HB & Blood and tissue \\
\hline Murali et al. 2014 & India & Asian & TaqMan & 449 & 445 & HB & Blood \\
\hline Liu et al. 2011 & China & Asian & PCR-RFLP & 101 & 102 & HB & $\begin{array}{c}\text { Oral mucosa } \\
\text { swabs }\end{array}$ \\
\hline Tsai et al 2011 & China & Asian & PCR-RFLP & 620 & 620 & PB & Blood \\
\hline Gomes et al. 2008 & Brazil & Mixed & PCR-RFLP & 80 & 80 & PB & $\begin{array}{c}\text { Oral mucosa } \\
\text { swabs }\end{array}$ \\
\hline Sathyan et al. 2006 & India & Asian & PCR-RFLP & 137 & 146 & HB & Blood and Tissue \\
\hline Holley et al. 2005 & Germany & Caucasian & PCR-RFLP & 155 & 174 & HB & Blood \\
\hline Nishimoto et al. 2004 & Brazil & Mixed & PCR & 135 & 147 & HB & Blood \\
\hline Wong et al. 2003 & China & Asian & PCR-SSCP & 93 & 70 & HB & Blood \\
\hline Zheng et al. 2001 & USA & Caucasian & PCR-SSCP & 248 & 233 & HB & Blood \\
\hline Matthias et al. 1998 & Germany & Caucasian & PCR-RFLP & 191 & 38 & HB & Blood \\
\hline
\end{tabular}

Table 2. CCND1 G870A gene polymorphism from eligible publications

\begin{tabular}{lccccccccc}
\hline \multirow{2}{*}{ Eligible Publications } & \multicolumn{4}{c}{ Controls } & \multicolumn{3}{c}{ Cases } & HWE $^{*}$ \\
\cline { 2 - 11 } & GG & GA & AA & MAF & GG & GA & AA & MAF & p-value \\
\hline Atac et al. 2014 & 20 & 56 & 26 & 0.529 & 13 & 55 & 15 & 0.512 & 0.303 \\
\hline Murali et al. 2014 & 110 & 206 & 126 & 0.518 & 121 & 188 & 132 & 0.512 & 0.161 \\
\hline Liu et al. 2011 & 45 & 29 & 27 & 0.410 & 23 & 43 & 36 & 0.563 & 0.000 \\
\hline Tsai et al 2011 & 365 & 155 & 565 & 0.592 & 84 & 323 & 213 & 0.604 & 0.000 \\
\hline Gomes et al. 2008 & 28 & 29 & 23 & 0.468 & 25 & 30 & 25 & 0.5 & 0.014 \\
\hline Sathyan et al. 2006 & 40 & 61 & 36 & 0.485 & 36 & 71 & 39 & 0.510 & 0.203 \\
\hline Holley et al. 2005 & 40 & 87 & 28 & 0.461 & 66 & 94 & 14 & 0.350 & 0.107 \\
\hline Nishimoto et al. 2004 & 40 & 69 & 26 & 0.448 & 53 & 68 & 26 & 0.408 & 0.698 \\
\hline Wong et al. 2003 & 17 & 49 & 27 & 0.553 & 15 & 36 & 19 & 0.528 & 0.523 \\
\hline Zheng et al. 2001 & 78 & 129 & 41 & 0.425 & 62 & 116 & 55 & 0.484 & 0.313 \\
\hline Matthias et 1. 1998 & 55 & 101 & 35 & 0.447 & 7 & 20 & 11 & 0.552 & 0.338 \\
\hline
\end{tabular}

*Hardy-Weinberg equilibrium

Table 3. Newcastle-Ottawa analysis Results

\begin{tabular}{lccc}
\hline \multirow{2}{*}{$\begin{array}{l}\text { First author and } \\
\text { year }\end{array}$} & \multicolumn{3}{c}{ Quality indicators } \\
\cline { 2 - 4 } & $\begin{array}{c}\text { Selec- } \\
\text { tion }\end{array}$ & $\begin{array}{c}\text { Compara- } \\
\text { bility }\end{array}$ & $\begin{array}{c}\text { Expo- } \\
\text { sure }\end{array}$ \\
\hline Atac (2014) & $* *$ & $*$ & $* * *$ \\
\hline Murali (2014) & $* *$ & $*$ & $* *$ \\
\hline Liu (2011) & $* *$ & $*$ & $* *$ \\
\hline Tsai (2011) & $* *$ & $*$ & $* *$ \\
\hline Gomes (2008) & $* * *$ & $*$ & $* * *$ \\
\hline Sathyan (2006) & $* *$ & $*$ & $* *$ \\
\hline Holley (2005) & $* *$ & $*$ & $* *$ \\
\hline Nishimoto (2004) & $* * *$ & $*$ & $* * *$ \\
\hline Wong (2003) & $* *$ & $*$ & $* *$ \\
\hline Zheng (2001) & $* *$ & $*$ & $* * *$ \\
\hline Matthias (1998) & $* * *$ & $*$ & $* *$ \\
\hline
\end{tabular}

models and the results indicated no significant publication bias for CCND1 G870A gene polymorphism (Supplementary Information - Fig SI1: overall; Fig SI2: Caucasian; Fig SI3: Asian) (Table 4: overall; Table 5: Caucasian; \& Table 6: Asian).

Heterogeneity assessment revealed significant heterogeneity in all the genetic models for overall risk. Therefore, we applied the random-effects model for the analysis (Table 4). Likewise, observable diversity was conceived in the four genetically different models (Caucasian: Table 5 and Asian population: Table 6). 
Table 4. Publication bias and heterogeneity Analysis, Overall population

\begin{tabular}{lccccccc}
\hline \multirow{2}{*}{ Comparison } & \multicolumn{3}{c}{ Egger's regression } & \multicolumn{3}{c}{ Heterogeneity } & \multirow{2}{*}{ Models } \\
\cline { 2 - 7 } & Intercept & $\mathbf{9 5 \%}$ Confidence Interval & p-value & Q-value & $\mathbf{P}_{\text {heterogeneity }}$ & $\mathbf{I}^{\mathbf{2}}$ (\%) & \\
\hline $\mathrm{A} / \mathrm{G}$ & 0.44 & -2.41 to 3.30 & 0.73 & 25.53 & 0.004 & 60.83 & Random \\
\hline $\mathrm{AA} / \mathrm{GG}$ & -1.02 & -3.93 to 1.88 & 0.44 & 29.96 & 0.001 & 66.63 & Random \\
\hline $\mathrm{AG} / \mathrm{GG}$ & -3.75 & -11.64 to 4.19 & 0.30 & 169.25 & 0.000 & 94.09 & Random \\
\hline $\mathrm{AA}+\mathrm{AG} / \mathrm{GG}$ & -2.18 & -7.26 to 2.89 & 0.35 & 76.19 & 0.000 & 86.86 & Random \\
\hline $\mathrm{AA} / \mathrm{AG}+\mathrm{GG}$ & 2.47 & -0.52 to 5.48 & 0.09 & 50.56 & 0.000 & 80.22 & Random \\
\hline
\end{tabular}

Table 5. Publication bias and heterogeneity Analysis, Caucasian Population

\begin{tabular}{lccccccc}
\hline \multirow{2}{*}{ Comparison } & \multicolumn{3}{c}{ Egger's regression } & \multicolumn{3}{c}{ Heterogeneity } & \multirow{2}{*}{ Models } \\
\cline { 2 - 7 } & Intercept & $\mathbf{9 5 \%}$ Confidence Interval & p-value & Q-value & $\mathbf{P}_{\text {heterogeneity }}$ & $\mathbf{I}^{\mathbf{2}(\%)}$ & \\
\hline $\mathrm{A} / \mathrm{G}$ & 0.39 & -23.35 to 24.13 & 0.94 & 14.63 & 0.002 & 79.49 & Random \\
\hline $\mathrm{AA} / \mathrm{GG}$ & -1.33 & -23.81 to 21.15 & 0.82 & 16.35 & 0.001 & 81.65 & Random \\
\hline $\mathrm{AG} / \mathrm{GG}$ & 1.95 & -7.71 to 11.62 & 0.47 & 5.13 & 0.160 & 41.62 & Fixed \\
\hline $\mathrm{AA}+\mathrm{AG} / \mathrm{GG}$ & 1.65 & -12.96 to 15.59 & 0.66 & 8.99 & 0.029 & 66.64 & Random \\
\hline $\mathrm{AA} / \mathrm{AG}+\mathrm{GG}$ & -3.82 & -26.33 to 18.68 & 0.54 & 14.37 & 0.002 & 79.12 & Random \\
\hline
\end{tabular}

Table 6. Publication bias and heterogeneity Analysis, Asian population

\begin{tabular}{|c|c|c|c|c|c|c|c|}
\hline \multirow{2}{*}{ Comparisons } & \multicolumn{3}{|c|}{ Egger's regression } & \multicolumn{3}{|c|}{ Heterogeneity } & \multirow{2}{*}{ Models } \\
\hline & Intercept & 95\% Confidence Interval & p-value & Q-value & $\mathbf{P}_{\text {heterogeneity }}$ & $I^{2}(\%)$ & \\
\hline A / G & 1.37 & -4.00 to 6.75 & 0.47 & 9.01 & 0.06 & 55.62 & Fixed \\
\hline $\mathrm{AA} / \mathrm{GG}$ & -0.32 & -6.77 to 6.11 & 0.88 & 10.21 & 0.03 & 60.83 & Random \\
\hline $\mathrm{AG} / \mathrm{GG}$ & -4.30 & -28.08 to 19.48 & 0.60 & 125.02 & 0.00 & 96.80 & Random \\
\hline $\mathrm{AA}+\mathrm{AG} / \mathrm{GG}$ & -2.22 & -16.65 to 12.20 & 0.65 & 48.76 & 0.00 & 91.79 & Random \\
\hline $\mathrm{AA} / \mathrm{AG}+\mathrm{GG}$ & 3.94 & -3.16 to 11.04 & 0.17 & 29.84 & 0.00 & 86.59 & Random \\
\hline
\end{tabular}

Association of CCND1 G870A gene polymorphism and overall $O C$ risk

Clinical genotype data retrieved from eleven eligible studies resulted in 2138 cases and 2311 controls. All the subjects were examined for the association between CCND1 G870A SNP and overall OC risk. No significant association was found between CCND1 G870A gene polymorphism and overall oral cancer risk in allele (A / G: $\mathrm{p}=0.0511 ; \mathrm{OR}=1.050,95 \% \mathrm{CI}=0.908$ to 1.215$)$, homozygous (AA / GG: $\mathrm{p}=0.387$; OR=1.151, $95 \% \mathrm{CI}=0.837$ to 1.581 ), heterozygous ( $\mathrm{AG}$ / GG: $\mathrm{p}=0.315 ; \mathrm{OR}=1.389,95 \% \mathrm{CI}=0.732$ to 2.636), dominant (AA+AG / GG: $\mathrm{p}=0.269$; $\mathrm{OR}=1.254,95 \% \mathrm{CI}=0.839$ to 1.875$)$ and reces- sive (AA / AG+GG: $p=0.677 ; \mathrm{OR}=0.932,95 \%$ $\mathrm{CI}=0.671$ to 1.296 ) genetic models (Fig 2).

\section{Ethnicitybased sub-group analysis.}

Sub-group analyses were then performed based on the ethnicity in Caucasian and Asian population. Caucasian sub-group exhibited inter-study heterogeneity, hence the model with randomized effect was selected for analysis. CCND1 G870A gene polymorphism and OC risk was not observed for any gene combination $(\mathrm{A} / \mathrm{G}$ : $\mathrm{p}=0.928$; $\mathrm{OR}=1.018,95 \% \mathrm{CI}=0.689$ to 1.504$)$, homozygous (AA / GG: $\mathrm{p}=0.976$; OR $=1.014,95 \%$ $\mathrm{CI}=0.406$ to 2.534), heterozygous (AG / GG: $\mathrm{p}=0.948 ; \mathrm{OR}=1.009,95 \% \mathrm{CI}=0.762$ to 1.336$)$, 


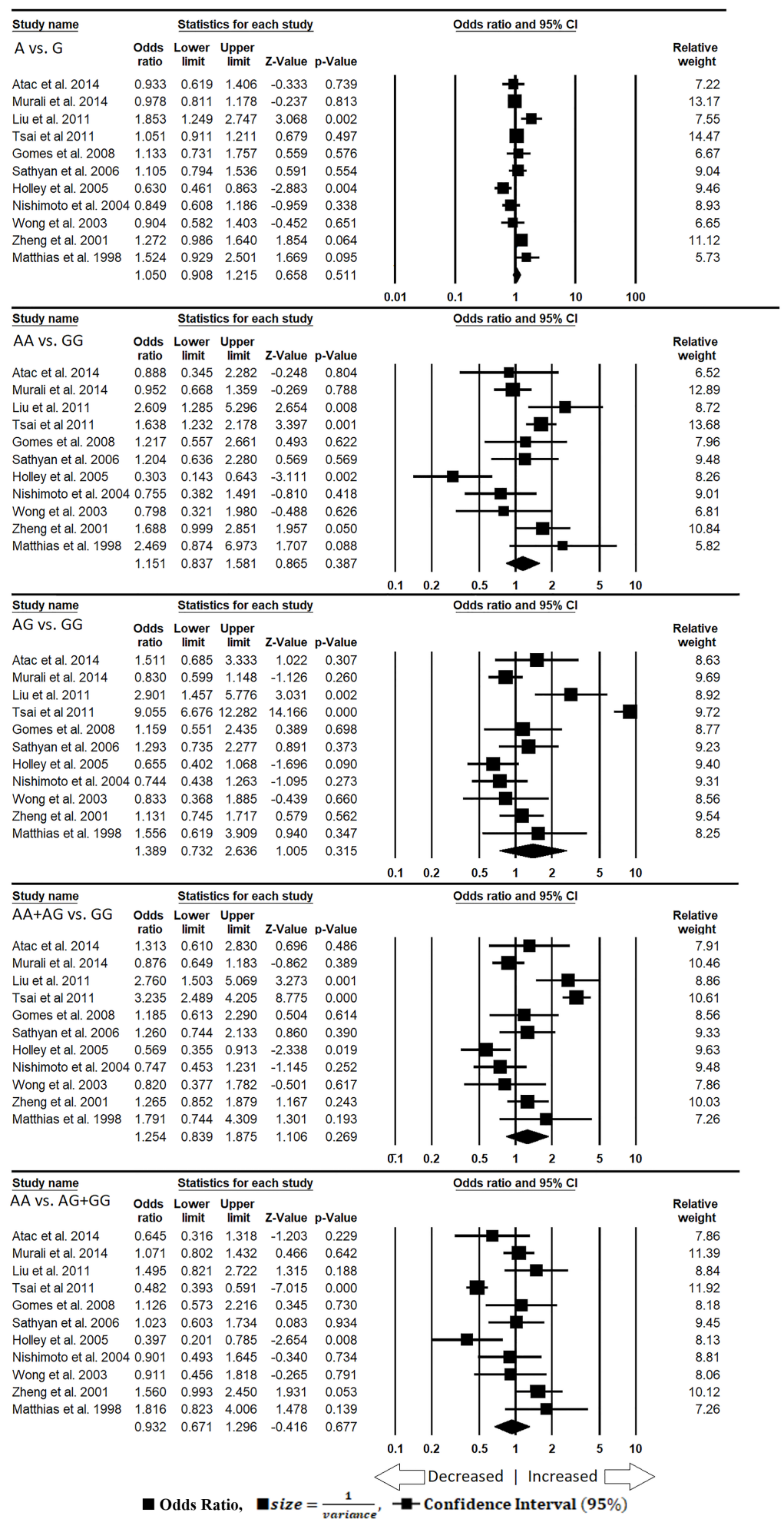

Fig. 2. CCND1 G870A SNP Forest plot for OC risk, 95\% CI (overall population). 
dominant (AA+AG / GG: $\mathrm{p}=0.783$; $\mathrm{OR}=1.074$, $95 \% \mathrm{CI}=0.646$ to 1.785 ) and recessive (AA / $A G+G G: p=0.843 ; \mathrm{OR}=0.931,95 \% \mathrm{CI}=0.459$ to 1.887) of Caucasian sub-group (Fig 3 ).

In the Asian population, the study between heterogeneity was observed in four genetic models. Thus, the meta-analysis was performed using random model, however the remaining one was performed using the fixed effects model. The combined results based on all the studies showed that allele (A / G: $\mathrm{p}=0.225 ; \mathrm{OR}=1.064$, $95 \% \mathrm{CI}=0.962$ to 1.177 ), homozygous (AA / GG: $p=0.122 ; \quad O R=1.331,95 \% \quad C I=0.926$ to 1.913), heterozygous (AG / GG: AA / GG: $\mathrm{p}=0.280 ; \mathrm{OR}=1.902,95 \% \mathrm{CI}=0.593$ to 6.097$)$, dominant $(\mathrm{AA}+\mathrm{AG} / \mathrm{GG}$ : $\mathrm{p}=0.201$; $\mathrm{OR}=1.544$, $95 \% \mathrm{CI}=0.794$ to 3.004 ) and recessive (AA / AG+GG: $\mathrm{p}=0.694 ; \mathrm{OR}=0.908,95 \% \mathrm{CI}=0.561$ to $1.470)$ genetic models failed to show any significant association between the cases and controls (Fig 4).

\section{Analysis of sensitivity}

Leave-one-out sensitivity analysis was performed to determine the effect of studies (separately, one at a time) and pooled ORs for CCND1 G870A gene polymorphism were derived for each case.

Leave-one-out sensitivity analysis was performed to determine if the overall analysis was effected by singular studies. The results of the sensitivity analysis revealed that no bias was caused by any specific singular study on the overall analysis (Supplementary Information Fig SI4: overall; Fig SI5: Caucasian; Fig SI6: Asian). This approves the credibility and reliability of the final inferences.

\section{Trial sequential analysis}

TSA was used to investigate the relevance of CCND1 G870A gene polymorphism with OC development risk. The dominant model was used to study this polymorphism. As we found, the cumulative Z-curve for this polymorphism did not surpass the monitoring boundary without achieving the required number of samples, hence it indicated that the cumulative evidence was inadequate and further trials were required for overall (Fig 5c) and sub-group analysis of Caucasian (Fig 5b) and Asian (Fig 5a) population.

\section{Discussion}

Genetic alterations occurring in important molecular pathways may play significant roles in cancer development together with environmental or lifestyle factors (37). It is vital to identify the molecular biomarkers as risk factors for better understanding of the pathogenesis of $\mathrm{OC}$ in order to improve the diagnostic accuracy, reduce the incidence rate, and help to plan suitable treatments strategies.

CCND1 is activated by Cyclin-dependent kinase 4 and 6 (CDK4 and CDK6) (38). Cyclin D1 catalyzes the phosphorylation of the tumor suppressor protein retinoblastoma (RB). The phosphorylation of $\mathrm{RB}$ releases the transcriptional factor E2F, which then activates a number of downstream genes necessary for cell cycle progression and transition from the G1 to S phase (39). Transition through G1 to S phase is an important checkpoint to prevent the replication of damaged DNA and allow DNA damage to be repaired (40). Earlier scientific studies have reported that alteration in the expression of proteins in the cell cycle is associated with malignant lesions that arise in the oral cavity $(41,42)$. Given the significance of control of the cell cycle for the maintenance of genomic integrity, it is conceived that common polymorphism in CCND1 gene may play an important biological role and initiate OC development.

The G870A polymorphism of CCND1 gene is associated with a splice site variation coding, which increases alternative splicing for two mRNA transcripts (15). The transcript ' $b$ ' is as- 


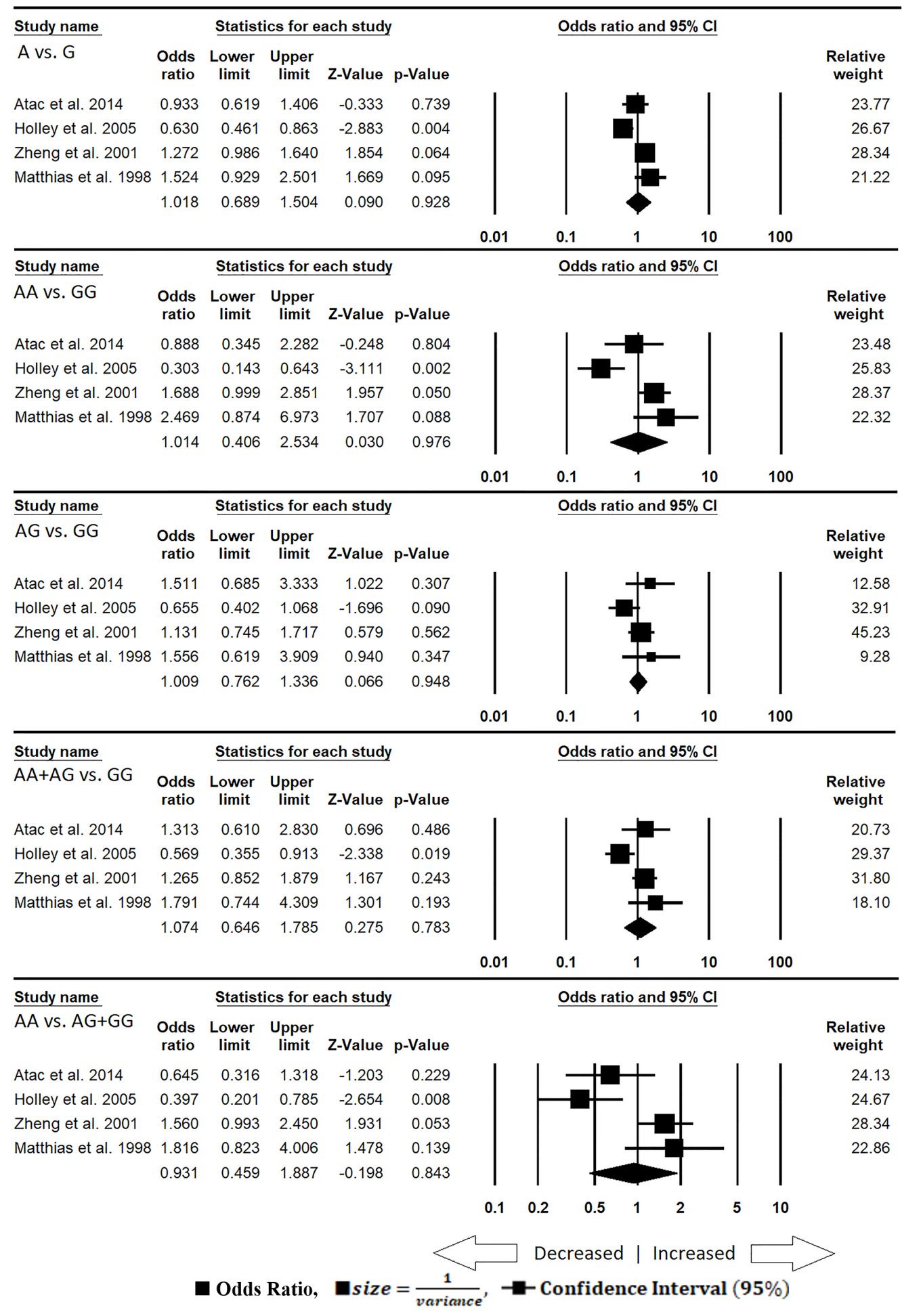

Fig. 3. CCND1 G870A SNP Forest plot for OC risk, 95\% CI (Caucasian population). 


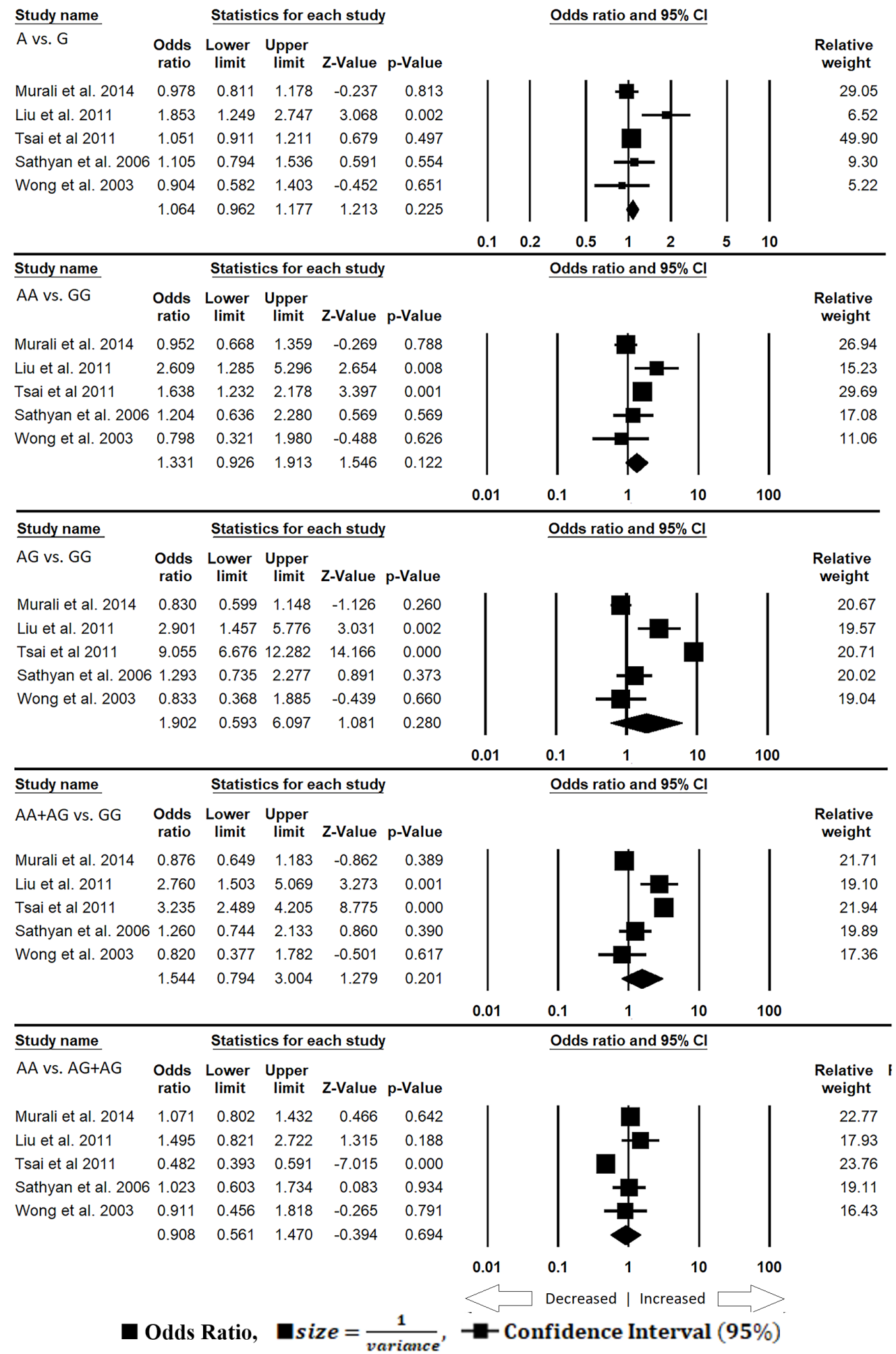

Fig. 4. CCND1 G870A SNP Forest plot for OC risk, 95\% CI (Asian population). 


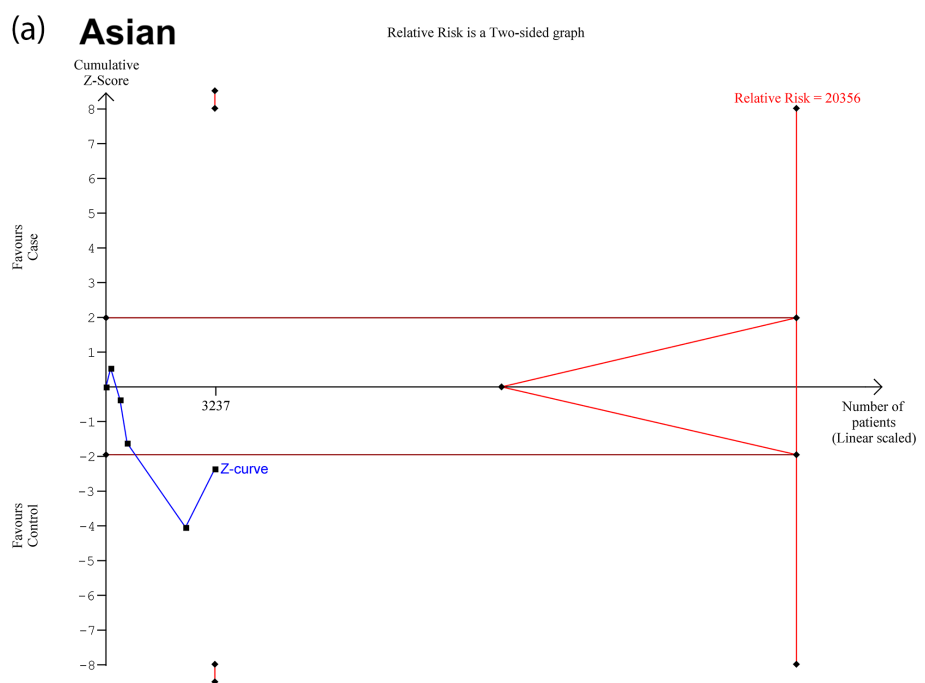

(b) Caucasian

Relative Risk is a Two-sided graph

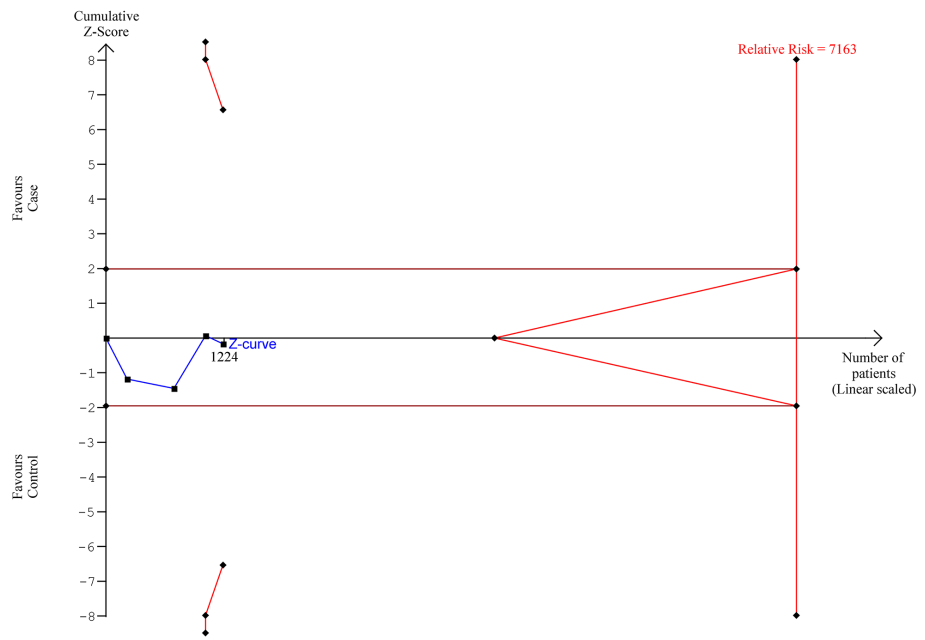

(c) Overall

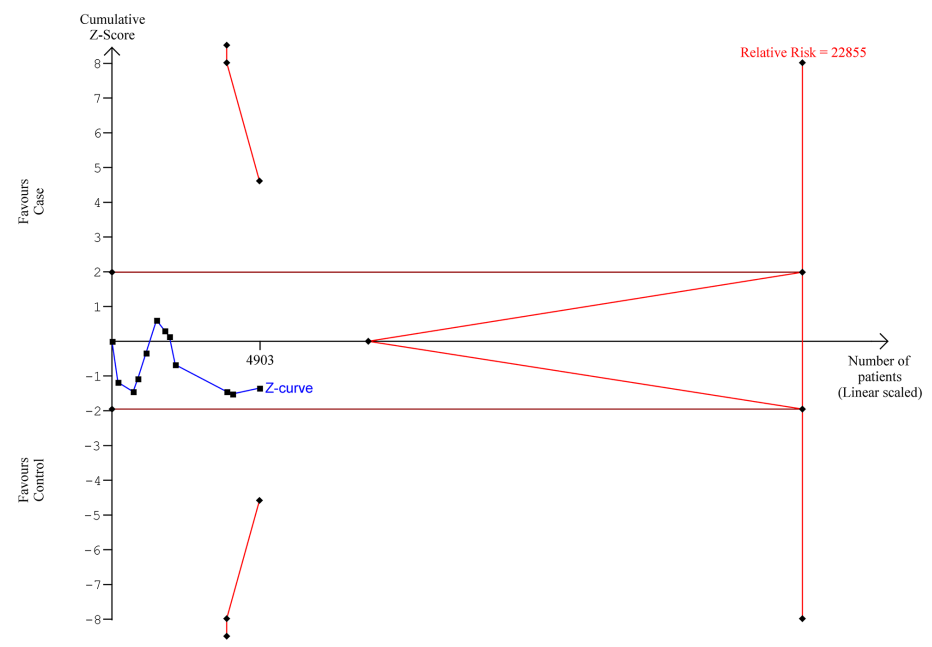

Fig. 5. TSA analysis of polymorphism in CCND1 G870A gene (dominant model), (a) Asian (b) Caucasian, (c) Overall population and $\mathrm{OC}$ risk. 
sociated with A allele, while $\mathrm{G}$ allele is associated with both transcript ' $a$ ' and transcript ' $b$ ' (15, 43). The encoded protein half-life for transcript ' $b$ ' was found to be more than that of transcript 'a'. It is suggested that cells overexpressing transcript 'a' promote entry into the cell cycle, whereas the transcript ' $b$ ' expressing cells are associated with cell cycle exit (43). Elevated presence of CCND1 may cause damaged DNA and associated genetic errors conveyed to daughter cells via G1-S transition due to premature cell cycle exit (44). The conventional approach of using individual case-control studies yields many basic elements of analytic complexity and can seriously underestimate the true sample size requirement. The present study meta-analyses the effect of eleven case control studies to discover any association between CCND1 gene SNP and $\mathrm{OC}$ risk. We observed no association risk of OC development linked with CCND1 G870A SNP based on overall population and subgroup analysis by ethnicity in Caucasian and Asian population. These results suggest that CCND1 G870A SNP might not contribute to the development of OC risk. The possible explanation is that CCND1 G870A SNP has different biological significance to various cancers, but not as a potential risk factor for OC development. The present findings correspond well with those of previous analyses $(45,46)$.

\section{Strengths and limitations}

As predicted earlier that etiology of OC is polygenic in nature, therefore, a single genetic variant is usually insufficient to predict the risk of this lethal disease. The current meta-analysis had a certain level of comprehensiveness, but still there were some limitations, which should be taken into consideration. In the present study, we detected significant heterogeneity which was accordingly minimized by applying the random-effects model. Random-effects model is generally used to consolidate the highly heterogeneous results to give more conservative and more precise results. It is worth noting that the results must be interpreted with caution, since our estimations regarding the sub-group analysis were based on the limited studies. Only published articles indexed in three reliable databases were included in our meta-analysis, therefore other relevant studies available in other databases might be omitted. These results are based on unadjusted OR and without gene environment interactions. A more precise analysis must be done using the data from individuals, allowing researchers to adjust for covariates including age, ethnicity, family history, environmental factors, and lifestyle. This being said, the present meta-analysis does promise valuable genotype to phenotype information. Foremost, all the eligible studies comprising the present meta-analysis followed the strict pre-set inclusion/exclusion criteria. Secondly, no bias was detected in the publications included in this meta-analysis, which suggests that the inferences drawn are resilient and reliable. The results of sensitivity analysis further vindicate our claim of strong and reliable inferences, since no significant effect on the pooled OR was observed for all the studies included in this analysis. Statistical analysis performed in the present analysis is as per the standard protocol prescribed for meta-analysis.

\section{Conclusions}

Overall, the current meta-analysis suggests that CCND1 G870A gene polymorphism is unlikely to be associated with the risk of OC. The importance of this polymorphism as a predictor of the risk of OC is probably very small and the screening utility of this genetic polymorphism in asymptomatic individuals might not be warranted.

\section{Funding Statement}

This research has been funded by Scientific Research Deanship at University of Ha'il - Saudi Arabia through Project Number RG-20011. 


\section{Acknowledgement}

The Authors acknowledge the support of Saudi Digital Library services provided by Hail University.

\section{Conflict of Interest}

The authors report no conflicts of interest.

\section{Data Availability Statement}

The Figures data used to support the findings of this study are included within the supplementary information file.

\section{References}

1. Ferlay J, Soerjomataram I, Dikshit R, Eser S, Mathers C, Rebelo M, Parkin DM, Forman D, Bray F. Cancer incidence and mortality worldwide: sources, methods and major patterns in GLOBOCAN 2012. Int J Cancer. 2015 Mar 1;136(5):E359-86. DOI: 10.1002/ijc.29210

2. Hajishengallis G, Lamont RJ. Beyond the red complex and into more complexity: the polymicrobial synergy and dysbiosis (PSD) model of periodontal disease etiology. Mol Oral Microbiol. 2012: 27:409-19. DOI: 10.1111/j.2041-1014.2012.00663.x

3. Atanasova KR, Yilmaz O. Looking in the Porphyromonas gingivalis cabinet of curiosities: the microbium, the host and cancer association. Mol Oral Microbiol. 2014: 29:55-66. DOI: 10.1111/omi.12047

4. Whitmore SE, Lamont RJ. Oral bacteria and cancer. PLoS Pathog. 2014: 10:e1003933. DOI: 10.1371/journal.ppat.1003933

5. Hooper SJ, Wilson MJ, Crean SJ. Exploring the link between microorganisms and oral cancer: a systematic review of the literature. Head Neck. 2009: 31:1228-39. DOI: $10.1002 /$ hed. 21140

6. Ahn J, Segers S, Hayes RB. Periodontal Disease, Porphyromonas Gingivalis (P. gingivalis) Serum Antibody Levels and Orodigestive Cancer Mortality. Carcinogenesis. 2012: 33:1055-8. DOI: 10.1093/carcin/bgs112

7. Tezal M, Sullivan MA, Hyland A, Marshall JR, Stoler D, Reid ME, Loree TR et al. Chronic periodontitis and the incidence of head and neck squamous cell carcinoma. Cancer Epidemiol Biomarkers Prev. 2009:
18:2406-12. DOI: 10.1158/1055-9965.EPI-09-0334

8. Ahn J, Chen CY, Hayes RB. Oral microbiome and oral and gastrointestinal cancer risk. Cancer Causes Cont. 2012: 23:399-404. DOI: 10.1007/s10552-011-9892-7

9. Han YW, Houcken W, Loos BG, Schenkein HA, Tezal M. Periodontal disease, atherosclerosis, adverse pregnancy outcomes, and head-and-neck cancer. Adv Dent Res. 2014: 26:47-55. DOI: 10.1177/0022034514528334

10. Bartkova J, Lukas J, Muller H, Strauss M, Gusterson B, Bartek J. Abnormal patterns of D-type cyclin expression and G1 regulation in human head and neck cancer. Cancer Res. 1995: 55:949-56.

11. Hohberger L, Wuertz BR, Xie H, Griffin T, Ondrey F. TNF-alpha drives matrix metalloproteinase-9 in squamous oral carcinogenesis. Laryngoscope. 2008: 118:1395-9. DOI: 10.1097/MLG.0b013e318174e09b

12. Jackson-Bernitsas DG, Ichikawa H, Takada Y, Myers JN, Lin XL, Darnay BG et al. Evidence that TNFTNFR1-TRADD-TRAF2-RIP-TAK1-IKK pathway mediates constitutive NF-kappaB activation and proliferation in human head and neck squamous cell carcinoma. Oncogene. 2007: 26:1385-97. DOI: 10.1038/ sj.onc. 1209945

13. Sherr CJ. Cancer cell cycles. Science. 1996:274(5293):1672-7. DOI: 10.1126/science.274.5293.1672

14. Witzel II, Koh LF and Perkins ND: Regulation of cyclin D1 gene expression. Biochem Soc Trans 2010: 38:217-22. DOI: 10.1042/BST0380217

15. Betticher DC, Thatcher N, Altermatt HJ, Hoban P, Ryder WD, Heighway J. Alternate splicing produces a novel cyclin D1 transcript. Oncogene. 1995:11(5):1005-11.

16. Soloman DA, Wang Y, Fox SR, Lambeck TC, Giesting $\mathrm{S}$, Lan Z, et al. Cyclin D1 splice variants. Differential effects on localization, RB phosphorylation, and cellular transformation. J Biol Chem. 2003 :278(16): 3033947.

17. Atac A, Riecke B, Assaf AT, Gröbe A, Friedrich RE, Heiland $\mathrm{M}$ et. al. Lack of evidence for predictive and prognostic value of cyclin D1 gene polymorphism CCND1 G870A for oral squamous cell carcinoma. Anticancer Res. 2014:34(1):235-8.

18. Murali A, Nalinakumari KR, Thomas S, Kannan S. Association of single nucleotide polymorphisms in cell cycle regulatory genes with oral cancer susceptibility. Br J Oral Maxillofac Surg. 2014:52(7):652-8. DOI: 10.1016/j.bjoms.2014.05.010 
19. Liu W, Zhu E, Wang R, Wang L, Gao L, Yang X, Liu T. Cyclin D1 gene polymorphism, A870G, is associated with an increased risk of salivary gland tumors in the Chinese population. Cancer Epidemiol. 2011:35(4):e12-7. DOI: 10.1016/j.canep.2010.11.001

20. Tsai MH, Tsai CW, Tsou YA, Hua CH, Hsu CF, Bau DT. Significant association of cyclin D1 single nucleotide polymorphisms with oral cancer in taiwan. Anticancer Res. 2011:31(1):227-31.

21. Gomes CC, Drummond SN, Guimarães AL, Andrade CI, Mesquita RA, Gomez RS. P21/WAF1 and cyclin D1 variants and oral squamous cell carcinoma. J Oral Pathol Med. 2008:37(3):151-6. DOI: 10.1111/j.16000714.2007.00604.x

22. Sathyan KM, Nalinakumari KR, Abraham T, Kannan S. Influence of single nucleotide polymorphisms in H-Ras and cyclin D1 genes on oral cancer susceptibility. Oral Oncol. 2006:42(6):607-13. DOI: 10.1016/j.oraloncology.2005.10.019

23. Holley SL, Matthias C, Jahnke V, Fryer AA, Strange $\mathrm{RC}$, Hoban PR. Association of cyclin D1 polymorphism with increased susceptibility to oral squamous cell carcinoma. Oral Oncol. 2005:41(2):156-60. DOI: 10.1016/j.oraloncology.2004.08.005

24. Nishimoto IN, Pinheiro NA, Rogatto SR, Carvalho AL, Simpson AJ, Caballero OL et al. Cyclin D1 gene polymorphism as a risk factor for squamous cell carcinoma of the upper aerodigestive system in non-alcoholics. Oral Oncol. 2004:40(6):604-10. DOI: 10.1016/j. oraloncology.2003.12.009

25. Wong YK, Lin SC, Chang CS, Tseng YH, Liu CJ, Lin $\mathrm{HC}$ et al. Cyclin D1 genotype in areca-associated oral squamous cell carcinoma. J Oral Pathol Med. 2003:32(5):265-70. DOI: 10.1034/j.16000714.2003.00131.x

26. Zheng Y, Shen H, Sturgis EM, Wang LE, Eicher SA, Strom SS et al. Cyclin D1 polymorphism and risk for squamous cell carcinoma of the head and neck: a case-control study. Carcinogenesis. 2001:22(8):1195-9. DOI: $10.1093 /$ carcin/22.8.1195

27. Matthias C, Branigan K, Jahnke V, Leder K, Haas J, Heighway $\mathrm{J}$ et al. Polymorphism within the cyclin D1 gene is associated with prognosis in patients with squamous cell carcinoma of the head and neck. Clin Cancer Res. 1998:4(10):2411-8.

28. Munafo MR, Flint J. Meta-analysis of genetic association studies. Trends Genet. 2004:20(9):439-44. DOI: 10.1016/j.tig.2004.06.014

29. Stang A: Critical evaluation of the Newcastle-Ottawa scale for the assessment of the quality of nonrandomized studies in meta-analyses. Eur J Epidemiol 2010: 25: 603-5. DOI: 10.1007/s10654-010-9491-z

30. Wu R, Li B. A multiplicative-epistatic model for analyzing interspecific differences in outcrossing species. Biometrics. 1999:2:355-65. DOI: 10.1111/j.0006341X.1999.00355.x

31. Der Simonian R, Laird N. Meta-analysis in clinical trials. Control Clin Trials. 1986:7:177-88. DOI: 10.1016/0197-2456(86)90046-2

32. Mantel N, Haenszel W. Statistical aspects of the analysis of data from retrospective studies of disease. J Natl Cancer Inst. 1959:4:719-48.

33. Egger M, Davey Smith G, Schneider M, Minder C. Bias in meta-analysis detected by a simple, graphical test. BMJ. 1997: 315:629-34. DOI: 10.1136/ bmj.315.7109.629

34. Wetterslev, J., Thorlund, K., Brok, J., Gluud, C. Trial sequential analysis may establish when firm evidence is reached in cumulative metaanalysis. J. Clin. Epidemiol. 2008:61: 64-75. DOI: 10.1016/j.jclinepi.2007.03.013

35. d Brok, J., Thorlund, K., Wetterslev, J., and Gluud, C. Apparently conclusive meta-analyses may be inconclusive-Trial sequential analysis adjustment of random error risk due to repetitive testing of accumulating data in apparently conclusive neonatal meta-analyses. Int. J. Epidemiol 2009: 38: 287-98. DOI: 10.1093/ije/dyn188

36. Turner, R. M., Bird, S. M., and Higgins, J. P. The impact of study size on meta-analyses: examination of underpowered studies in Cochrane reviews. PLoS ONE 2013: 8: e59202. DOI: 10.1371/journal.pone.0059202

37. Wilson S, Jones L, Couseens C, Kathi H. The links between environmental factors, genetics, and the development of Cancer. Cancer and the Environment: Gene-Enviroment Interaction. National Academies Press (US), 2002.

38. Morgan, David O. Principles of CDK regulation. Nature 374, no. 6518 (1995): 131-4. DOI: 10.1038/374131a0

39. Dowdy, Steven F., Philip W. Hinds, Kenway Louie, Steven I. Reed et al. Weinberg. Physical interaction of the retinoblastoma protein with human D cyclins. Cell 1993:73(3): 499-511. DOI: 10.1016/00928674(93)90137-F

40. Donnellan, R., and R. Chetty. Cyclin D1 and human neoplasia. Molecular Pathology 1998):51:1. DOI: 
10.1136/mp.51.1.1

41. Schoelch ML, Regezi. Cell cycle proteins the development of oral squamous cell carcinoma. Oral Oncol 1999: 35:333-42. DOI: 10.1016/S1368-8375(98)00098-0

42. Todd R, Hind PW, Munger K, Rustgi AK, Cell cycle dysregulation in oral cancer. Crit Rev Oral Biol Med 2002:13:51-61. DOI: 10.1177/154411130201300106

43. Sawa H, Ohshima TA, Ukita H, Murakami H, Chiba Y, Kamada H, et al. Alternatively spliced forms of cyclin D1 modulate entry into the cell cycle in an inverse manner. Oncogene. 1998:16(13):1701-12. DOI: 10.1038/ sj.onc. 1201691
44. Hall M, Peters G. Genetic alterations of cyclins, cyclin-dependent kinases, and Cdk inhibitors in human cancer. Adv Cancer Res.1996:68:67-108. DOI: 10.1016/S0065-230X(08)60352-8

45. Wang L, Wang Z, Gao X, Li X, Sun X, Liu C. Association between Cyclin D1 polymorphism and oral cancer susceptibility: a meta-analysis. Tumour Biol. 2014:35(2):1149-55. DOI: 10.1007/s13277-013-1154-8

46. Wang W, Zhao Y, Yang J, Lin B, Gu H, Cao X et al. Cyclin D1 polymorphism and oral cancer: a meta-analysis. Mol Biol Rep. 2013:40(1):87-95. DOI: 10.1007/ s11033-012-2025-X 